\title{
Laboreal
}

Volume 15 N$^{\circ} 2$ | 2019

Varia

\section{Enfoque clínico de las relaciones entre salud y trabajo : contribuciones y desafíos}

Abordagem clínica das relações entre saúde e trabalho : contribuições e desafios Approche clinique des relations entre santé et travail : contributions et défis Clinical approach to the relationships between health and work: contributions and challenges

\section{Andrea Pujol y María Inés Gutiérrez}

\section{OpenEdition}

\section{Journals}

Edición electrónica

URL: http://journals.openedition.org/laboreal/15506

DOI: 10.4000/laboreal. 15506

ISSN: 1646-5237

\section{Editor}

Universidade do Porto

\section{Referencia electrónica}

Andrea Pujol y María Inés Gutiérrez, «Enfoque clínico de las relaciones entre salud y trabajo :

contribuciones y desafíos », Laboreal [En línea], Volume 15 №2 | 2019, Publicado el 01 diciembre 2019, consultado el 17 abril 2020. URL : http://journals.openedition.org/laboreal/15506 ; DOI : https:// doi.org/10.4000/laboreal.15506

Este documento fue generado automáticamente el 17 abril 2020.

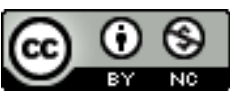

Laboreal está licenciado com uma Licença Creative Commons - Atribuição-NãoComercial 4.0 Internacional. 


\section{Enfoque clínico de las relaciones entre salud y trabajo: contribuciones y desafíos}

Abordagem clínica das relações entre saúde e trabalho : contribuições e desafios Approche clinique des relations entre santé et travail : contributions et défis Clinical approach to the relationships between health and work: contributions and challenges

\section{Andrea Pujol y María Inés Gutiérrez}

\section{NOTA DEL EDITOR}

Manuscrito recibido en junho/2019

Aceptado tras peritaje outubro/2019

\section{La problemática del trabajo contemporáneo y la salud}

1 La salud laboral constituye un campo de investigación específico que ha ido ganando en importancia en los estudios del trabajo en nuestro país. Desde diversas perspectivas teóricas se realizan esfuerzos significativos por conocer y comprender las vinculaciones entre salud y trabajo, la incidencia de las condiciones de trabajo en la emergencia de enfermedades profesionales, el estudio de la legislación y políticas de protección, y en los últimos años la importancia que adquieren los riesgos psicosociales en la salud de los trabajadores.

2 La agenda de los problemas del trabajo en nuestro país, al igual que en el resto de los países latinoamericanos, pone en foco la relación entre calidad del trabajo y salud laboral. Desde nuestra perspectiva, la calidad de trabajo se define al menos 
parcialmente en función de las posibilidades de construcción de sentido, de movilización subjetiva, que ofrece a los sujetos la organización del trabajo. La organización del trabajo propicia en mayor o menor medida la configuración de un trabajo de calidad, que satisfaga necesidades y que posibilite el desarrollo de los trabajadores, lo que implica la capacidad de sujetos y grupos de accionar cotidianamente en la búsqueda de un balance adecuado entre trabajo y vida.

3 En un contexto en el que casi un tercio de la población activa accede a empleos fragilizados, de baja remuneración y baja productividad, y en el que se produce una importante reconfiguración en la organización de las actividades y los oficios y la emergencia de empleo atípico, las nuevas formas de organización del trabajo traen consigo componentes importantes de intensificación del ritmo de trabajo, la paradoja de la prescripción de la participación, la sobre-responsabilización de los trabajadores sin contrapartidas que lo justifiquen, la falta de reconocimiento de los logros y en muchos casos también el deterioro de las condiciones y medio ambiente de trabajo como consecuencia de la reducción de costos o de la complejidad de los procesos de tercerización.

4 Las consecuencias de estas prácticas se evidencian tanto a nivel de la salud física como en el plano de la subjetividad, en el sufrimiento psíquico asociado a las condiciones en las que se trabaja actualmente, particularmente en lo relativo a la sensación de que "cuidar" el empleo supone aceptar -y en muchos casos naturalizar- exigencias y condiciones insatisfactorias, la vivencia de que progresar $u$ obtener mejoras en la propia carrera, desarrollarse en el trabajo, supone afrontar y resolver un conjunto importante de contradicciones individuales y colectivas.

5 La articulación salud y trabajo exige actualmente reconocer qué concepciones de empleo/trabajo/actividad y qué concepciones de sujeto permiten dar cuenta de la relación entre trabajo y subjetividad y cómo esta díada puede dar cuenta de la problemática de la salud laboral, a partir de una mejor comprensión de las contradicciones y las tensiones que marcan las trayectorias singulares y colectivas de los trabajadores y de cómo el bienestar y el sufrimiento que el trabajo implica se encarnan en sujetos y grupos social e históricamente situados.

6 Estas inquietudes tienen su anclaje en un estudio sobre las relaciones entre calidad del empleo, salud laboral y subjetividad que orientan una parte de nuestra producción (Pujol, 2018; Gutiérrez, 2018). Su desarrollo exigió el análisis previo de las contribuciones que dominan las investigaciones sobre salud laboral en Argentina, con el fin de resignificar la singularidad de los aportes de la perspectiva clínica. En dicho estudio, se analizaron las contribuciones teóricas y empíricas más divulgadas en el país, que incluyó algunos textos fundamentales y revisiones históricas para dar cuenta de los abordajes con mayor arraigo en el país y de comunicaciones y artículos científicos para la producción más reciente, que permiten valorar las concepciones puestas en juego, aunque todavía no se cuente con estados del arte de producción local.

7 En este marco, este escrito presenta en primer término las contribuciones sobre las Condiciones y medio ambiente de trabajo (СуMAT) y los abordajes ecológicos, como un conjunto de aportes que ponen en juego la noción de riesgo psicosocial. En segundo momento retomamos la perspectiva centrada en los procesos de subjetivación que al integrar los aportes de las clínicas del trabajo adopta en nuestro país un carácter emergente. A modo de cierre planteamos algunos desafíos que -a nuestro juicio- 
pueden ampliar las posibilidades de la perspectiva clínica en la investigación y la intervención en el campo de la salud laboral.

\section{La perspectiva de las CYMAT como abordaje institucional}

Entre los abordajes con más arraigo en los estudios sobre la salud laboral en Argentina, se encuentran las contribuciones de la sociología del trabajo y el empleo que en la década del 80 constituyeron un enfoque renovador que se plasmó en la adopción de la noción de condiciones y medioambiente de trabajo, conocidas bajo la sigla CyMAT (Neffa \& Del Bono, 2016). Esta noción incluye múltiples dimensiones que no sólo se relacionan con el cuidado de la salud de los trabajadores y la resolución de conflictos derivados de esta problemática, sino también con la preocupación por las actividades de prevención tendientes a reducir o limitar los riesgos laborales, antes asociados a la idea de compensación.

Hasta ese momento, en línea con las contribuciones clásicas de la seguridad y la higiene en el trabajo, los accidentes y enfermedades profesionales adoptaban el carácter de hechos fatales e imprevisibles, determinados necesariamente por la tecnología y la naturaleza misma del trabajo, ante lo que solamente cabía prever las indemnizaciones para reparar ex-post el daño. En consecuencia, no tenían lugar las medidas con finalidades de prevención, dado que las necesidades urgentes de la producción llevaban a concentrar la atención del proceso productivo en la situación presente y cotidiana, sin analizar los antecedentes de los incidentes y accidentes pasados.

Desde una fuerte referencia a la ergonomía de origen francófono, las CyMAT dan cuenta de una visión macroergonómica y holista de los puestos de trabajo, acentuando su influencia en la eficiencia de la organización, en tanto su implementación conlleva a mejoras de competitividad. En este sentido, cabe señalar que la ergonomía francófona constituye un aporte relevante en la medida en que sus desarrollos han contribuido tanto a fortalecer el enfoque de las CyMAT como el de la clínica del trabajo, principalmente a partir de un doble énfasis : la importancia otorgada a la distinción entre tarea y actividad, y el privilegio otorgado al sujeto, a través de su principio de búsqueda de la adaptación del trabajo al hombre.

11 Según el modelo analítico más difundido en nuestro país formulado por el CEIL (Neffa, 1995), las dimensiones contempladas en las CyMAT son determinadas por las características de los procesos de trabajo, y si bien enfatizan su importancia en el bienestar del trabajador, la perspectiva que se adopta para su definición es claramente organizacional. En este sentido, las buenas condiciones de trabajo impactarían en el incremento de la productividad, el mejoramiento de la calidad de bienes y servicios producidos, en la reducción de costos y en el mayor compromiso de los trabajadores.

Según Neffa, "Las condiciones y medio ambiente de trabajo (CYMAT) están constituidas por los factores socio-técnicos y organizacionales del proceso de producción implantado en el establecimiento (o condiciones de trabajo) y por los factores de riesgo del medio ambiente de trabajo" (Neffa, 1995, p.5). Ambos grupos de factores constituyen las exigencias, requerimientos y limitaciones del puesto de trabajo, que integran el trabajo prescripto y que impactan de modo directo en la salud de los trabajadores. En este enfoque, se adoptó originalmente la noción de salud provista por 
los trabajos de la Organización Mundial de la Salud, en los que se la entiende como ausencia de enfermedad y como un estado óptimo de bienestar físico, mental y social. Si bien esta noción ha sido posteriormente puesta en discusión, sigue vigente en gran parte de los estudios que adoptan este enfoque.

La contribución de esta perspectiva ha sido especialmente fructífera, porque puso en agenda de las empresas, organismos públicos y sindicatos la problemática de las condiciones y medio ambiente de trabajo como componentes clave de la salud laboral y logró cierto arraigo en los modelos de gestión de la fuerza de trabajo. Además, el carácter sociotécnico, organizacional, interdisciplinario y con foco en la gestión posibilitó el desarrollo de un conjunto de herramientas válidas para intervenir en diferentes contextos ocupacionales en el plano de la prevención de los riesgos.

Estas intervenciones operan principalmente sobre el trabajo prescripto y con la participación funcional de los trabajadores a partir de la ingeniería de los puestos y de estrategias educativas que promueven el desarrollo de la organización, propiciando un circuito virtuoso entre productividad y salud. En esta concepción, la noción de actividad como espacio de producción de sentido y como soporte de los procesos reflexivos sobre el género profesional resulta restringida. En la misma línea, si bien se utilizan estrategias de ergonomía participativa en los procesos de análisis y en las instancias formativas, la consideración de lo colectivo se restringe al plano de la participación funcional de los equipos y a la acción colectiva organizada en los procesos de negociación, sin que pueda capitalizarse el potencial que la clínica del trabajo otorga a esa categoría.

Estos rasgos remiten a que la perspectiva de las CyMAT en Argentina conserva en gran medida su formulación original sin que haya sido posible capitalizar algunas discusiones teóricas relevantes. En primer término, integrar las discusiones emergentes del avance de las ergo-disciplinas que han permitido el esclarecimiento de distintas dimensiones de la actividad y sus determinantes, en las que la incorporación de la dimensión subjetiva y la producción de sentido adquieren un rol clave.

En segundo lugar, la mirada institucionalista en la que se inscribe el abordaje de las Cymat enfatiza en la visión del trabajador como sujeto de derechos y asimila el trabajo a la idea de empleo clásico. En este marco, ante la emergencia del trabajo no clásico (De la Garza-Toledo, 2009) y la extensión de la precarización del empleo a las experiencias de trabajo (Paugam, 2006) se hacen evidentes los límites que puede encontrar este abordaje en el marco de un proceso de permanentes transformaciones y novedades de las actividades y la necesidad de asumir una actitud de vigilia permanente en la identificación y prevención de los riesgos (Neffa, 2015).

\section{Los abordajes ecológicos}

En la línea de las contribuciones de la psicología centradas en la relación factorrespuesta, los estudios del trabajo que se apoyan en una mirada ecológica, con anclaje en teorías sociocognitivas y socioconstruccionistas, constituyen el abordaje más internacionalizado de los que pueden encontrarse en las investigaciones de nuestro país.

Desde esta perspectiva, se desarrollan importantes contribuciones en Argentina sobre la violencia en el trabajo, el acoso laboral y el bullyng (Cebey \& Ferrari, 2010 ; Ferrari, 
Filippi, \& Trotta, 2011; Ferrari, Filippi, Novo, Sicardi, \& Trotta, 2013). Estas investigaciones se apoyan en análisis cuantitativos a través de la aplicación de cuestionarios al personal -en muchos casos de diversas jerarquías- de empresas y organismos, públicos y privados, dando continuidad de modo consistente a otros estudios internacionales con metodologías análogas. La consideración central de este abordaje son los sistemas de relaciones que se generan en los ambientes organizacionales, particularmente analizados como sistemas de autoridad y poder que estructuran tramas de relación asimétricas. En este marco, los hallazgos de estas investigaciones permiten el desarrollo de instrumentos de intervención entre los que se destacan los protocolos de abordaje, que resultan exitosos a la hora de regular las intervenciones sobre situaciones de violencia laboral y otros fenómenos morbosos de índole psicosocial particularmente asociados al trabajo contemporáneo.

En este marco, las intervenciones derivadas de este enfoque pueden inscribirse en la perspectiva del desarrollo organizacional, aunque en los últimos años han incorporado contribuciones de la psicología positiva y con ello tienden a propiciar un abordaje más centrado en el individuo que en los sistemas de relación. Esto deriva en la implementación de estrategias de intervención que se orientan a maximizar los recursos adaptativos de las personas y las capacidades socializadoras de la gestión. En estas aproximaciones, las estrategias de intervención enfatizan en la respuesta individual de los trabajadores ante los riesgos a partir del dominio de sus emociones y de la apelación a recursos personales y apoyos externos para afrontar las exigencias del trabajo. En contrapartida, este enfoque soslaya la necesidad de intervenir sobre los procesos de trabajo y su organización o poner en discusión los modos de disciplinamiento que se inscriben en la relación laboral entendida como institución.

El énfasis alternativo "en el ambiente" (los sistemas de relaciones) y "en la persona" (los recursos/fragilidades de los sujetos) de los abordajes hasta aquí mencionados tiene como principal fortaleza su alcance descriptivo -aunque no necesariamente heurísticoque permite la intervención desde la gestión para mitigar el efecto de las exigencias del trabajo en los operadores, con miras a maximizar -o al menos mantener invariante- la productividad, siempre desde una visión de adaptación del sujeto al universo de las prescripciones.

21 Desde la perspectiva clínica, aunque amplían la comprensión y tratamiento de las relaciones entre salud y trabajo, estas contribuciones proponen una mirada a-histórica que invisibiliza al trabajo como actividad, el carácter estructurante que adquiere la organización del trabajo en la salud en general y su expresión en los otros dominios de actividad. El papel de los colectivos y su poder para disputar en el campo político por un trabajo saludable a partir de procesos de resignificación y debate de las prescripciones es también relegado. Es posible señalar además que su carácter individualizante respecto a los recursos del trabajador lleva incluso a culpabilizar a quien no logra adaptarse y negando que la vulnerabilidad es constitutiva del sujeto.

Tanto en la revisión de la perspectiva de las CyMAT como en lo referido a los abordajes ecológicos, se hace necesario discutir la noción de riesgos -que adquiere un lugar central en estas propuestas- y del papel de los sujetos y colectivos a la hora de identificarlos y actuar sobre ellos, más allá de la intervención que le cabe a los sistemas de gestión sobre los ambientes de trabajo. En este desafío, resultan significativas las contribuciones de los estudios críticos del management que centran su discusión en la definición de los sistemas de relaciones y las identidades laborales. 


\section{Discusión clínica sobre la noción de riesgo}

La noción de riesgos vertebra gran parte de los estudios de salud y trabajo en Argentina, presente desde mediados de la década del ' 80 en las contribuciones de las CyMAT y el análisis de las enfermedades profesionales (Kohen, 1997) referenciando principalmente la problemática de la salud física y -en la última década- poniendo en foco la salud psíquica con la discusión y análisis de modelos de análisis específicos para los Riesgos Psicosociales en el Trabajo (Neffa, 2015 ; Henry \& Neffa, 2019).

Desde la perspectiva clínica el núcleo de la discusión sobre el enfoque de riesgos enfatiza en los supuestos que permiten considerar la dimensión psicosocial en un pie de igualdad respecto de otros factores y propiciar así una mirada reduccionista sobre tres cuestiones : la complejidad de las relaciones de sujetos y colectivos en la dinámica de la actividad, la noción de salud y la implicación mutua entre salud física y psíquica; lo psicosocial y la subjetividad.

Desde el punto de vista psicosocial, distintos abordajes comparten la idea de que la noción de riesgo resulta un concepto polisémico : puede referirse por una parte a los factores de riesgo y por otra a los daños que estos provocan sobre la salud, que pueden ser físicos, cognitivos o emocionales. Las dos dimensiones están fuertemente ligadas, y con frecuencia pueden confundirse. Por otra parte, esta imprecisión se juega tanto en relación al concepto de factor como a la noción de lo psicosocial en juego, debido a que finalmente lo psicosocial se establece de modo tan genérico que termina abarcándolo todo: condiciones externas al trabajador, el trabajador mismo, el estrés y sus repercusiones sobre la salud.

Las definiciones utilizadas por la Organización Internacional del Trabajo y la Organización Mundial de la Salud caracterizan a lo psicosocial como factores, es decir como un conjunto de elementos que se encuentran alrededor del trabajador y que pueden desencadenar algún posible daño en su salud. No se discute su origen y se considera que los riesgos psicosociales coexisten con los otros en la misma medida y con el mismo peso. Tampoco se discute el concepto mismo de factor y suele referirse a estos como riesgos o agentes. En este marco, lo psicosocial se asume en tres niveles: como condiciones causales, como procesos que median (el caso del estrés) y también en términos de las consecuencias (los daños a la salud). enfatice hoy en la carga emocional del trabajo, poco considerada en los estudios clásicos, dado que históricamente se atendió prioritariamente a las cargas físicas y mentales del trabajo desde una perspectiva psicofisiológica, lo que contribuyó a clarificar y comprender problemáticas clásicas como la fatiga y el estrés. Sin embargo, la ausencia de una mirada clínica en el análisis de la carga física y mental, invisibilizó al cuerpo como expresión primera de la subjetividad y con ello la dimensión emocional.

En este sentido, la organización del trabajo actual exige con frecuencia -y con mayor énfasis en el sector servicios- que el trabajador realice un esfuerzo significativo destinado a controlar y modelar sus emociones, para afrontar la actividad misma o para incidir en el comportamiento de otros. Además, actualmente se enfatiza en que cuando la naturaleza del trabajo implica actuar sobre otros en situación de sufrimiento (trabajo de cuidado, por ejemplo) la necesidad de control emocional se incrementa. En la 
mayoría de los ambientes de trabajo, no se actúa preventivamente creando las condiciones o los dispositivos que favorezcan que los trabajadores puedan manifestar o expresar las emociones y procesar cognitivamente la carga emocional ligada a la tarea, por lo que finalmente esta tiende a mantenerse oculta y volverse un componente fuertemente implicado en el sufrimiento en el trabajo y con efectos en la salud física.

Desde la perspectiva clínica, Yves Clot opone los riesgos psicosociales a los recursos psicológicos y sociales que son propios de los trabajadores y que han surgido del oficio como instrumentos de trabajo. Esta idea opera como disparador para cuestionar el enfoque de riesgos y proponer una visión del trabajador como un sujeto emocionalmente implicado y por ello activo respecto de los riesgos en el trabajo. El autor sostiene que los cambios impuestos por la organización del trabajo, las empresas y el estado han complicado y hasta deteriorado el "trabajo de organización" que los trabajadores tenían el hábito de realizar. En línea con algunos supuestos de la psicodinámica del trabajo, Clot afirma que la responsabilidad que se debe imputar a los dirigentes actuales no es la de querer modificar las organizaciones cuando lo necesitan sino más bien de hacerlo apropiándose del trabajo de organización que realizan los asalariados para lograr trabajar correctamente, dado que los trabajadores cultivan los recursos psicológicos y sociales de su oficio como instrumentos de trabajo (Clot, 2010).

Retomando contribuciones del ergónomo Daniellou, Yves Clot (2010) refiere también a que suele hablarse de los riesgos psicosociales como si se tratara de una nube tóxica que planea sobre la empresa o algunos de sus componentes, que afecta a los asalariados primero por supuesto a aquellos que por sus características personales son más frágiles. Según este modelo la atmósfera está contaminada y los más sensibles van a tener problemas. Las medidas que se pueden tomar al respecto se deducen de la imagen del modelo: se pueden hacer relevamientos previos de la atmósfera (valorar el entorno psicosocial), se pueden hacer dosajes a los asalariados de esa toxicidad para evaluar el riesgo que corren, se puede montar un sistema de señalización mutua (alertar cuando un colega comienza a no estar bien o se lo juzga frágil), se puede intentar desintoxicar a los que ya han sido afectados, por ejemplo ofreciéndoles un soporte psicológico financiado por la empresa. Por todos los medios lo que se tiende a hacer es que la nube no gane demasiadas víctimas, sobre todo aquellas cuya fragilidad intrínseca podría llevarlos a la tentativa de suicidio o aquellos efectos que son fuertemente perjudiciales para la organización y para la imagen de la marca.

31 El autor busca poner de relieve que esta visión tóxica deriva del modelo médico cientificista y de un paradigma en ergonomía ya superado, en el que la observación de la actividad se plasma en la medición para que pueda ser poco refutable y que por su supuesta neutralidad resulte en una señal de alerta para todos los sectores interesados en la naturaleza e intensidad de los riesgos evaluados. Esta mirada es la que se orienta a la búsqueda de un estándar internacional que nos lleve a un indicador global de riesgos en torno al cual plantear los protocolos. Así, el riesgo psicosocial queda asimilado a un riesgo tóxico y desemboca en una evaluación experta de la intensidad o consecuencia de los peligros, todo esto garantizado por la neutralidad de las mediciones.

Como hemos sostenido en otro lugar (Pujol, Gutiérrez, \& Barnes ; 2015) en el enfoque de riesgos la experiencia vivida queda fuera, aun cuando se trata de una prueba tangible de la salud, lo que implica una contradicción para la mirada clínica. Se buscan o investigan de antemano los impactos negativos e incluso la enfermedad, lo que es una elección. Saben que es más fácil para el que contesta pensar en lo que habría que 
cambiar que darse cuenta de lo que ha hecho, de lo que ha logrado gracias a los saberes construidos y sedimentados en el género profesional. En este sentido, en el marco de la clínica de la actividad es posible comprender que ciertas emociones sean tóxicas, pero las emociones son también socias en la acción de la transformación colectiva de las situaciones de trabajo, tienen virtud dinamógena en el plano psicológico y sabemos que el afecto es la fuente de la vitalidad psíquica y corporal.

En los términos expuestos, esta discusión de la noción de riesgo permite poner en valor al enfoque clínico como un resquicio para pensar la actividad de trabajo desde una perspectiva ampliada y las dinámicas de implicación de los sujetos y colectivos en la conquista de la salud como procesos emancipatorios, por oposición a las miradas en la que lo social limita las posibilidades de los sujetos/colectivos o a aquellas que proponen un reduccionismo psicologista.

En el marco de los desarrollos expuestos, entendemos que las contribuciones de la clínica del trabajo, de larga tradición en los países francófonos, se constituyen progresivamente en una mirada alternativa que enriquece el análisis de las relaciones del trabajador con la actividad, su inscripción institucional y colectiva y el lugar de la gestión del contexto organizacional y sus riesgos.

\section{Contribuciones de la clínica del trabajo en Argentina}

La perspectiva clínica se inscribe en Argentina en las tradiciones de la psicología social crítica y promueve un enfoque centrado en la subjetividad como producción social. Esta perspectiva articula contribuciones de sistemas teóricos en sintonía : la psicosociología y la sociología clínica, la psicodinámica del trabajo, la clínica de la actividad y la ergología. Desde estos referenciales teóricos, la temática salud-trabajo es abordada considerando su inscripción sociohistórica, incluyendo tanto el sufrimiento y los impactos negativos del trabajo en la salud como las posibilidades de afrontamiento de que disponen los sujetos y las potencialidades terapéuticas de las actividades de trabajo.

En este marco, los estudios locales colocan en primer plano algunos supuestos relevantes : la concepción clínica de la salud articulada a los procesos de subjetivación y a una mirada crítica del trabajo contemporáneo y la noción de actividad como soporte de la movilización subjetiva y su potencial transformador. En este marco nos interesa recuperar aquí algunos conceptos presentes en esta línea de investigaciones que se plantean como un resquicio para pensar la problemática de la salud.

En el marco del análisis de problemáticas diversas como las condiciones en que se despliega el trabajo de cuidado y las situaciones de violencia que atraviesa el personal de salud del sector público (Wlosko \& Ros, 2009; Wlosko \& Ros, 2012 ; BorgeaudGarciandía, 2016) algunos estudios locales de inscripción en la psicología social crítica y en sociología del trabajo, recuperan las consideraciones sobre la salud planteadas por la psicodinámica del trabajo y ponen en discusión la lógica de disciplinamiento del trabajo contemporáneo poniendo de relieve las marcas de la dominación, sus efectos de sufrimiento y también las capacidades colectivas de afrontamiento, enfatizando en la problemática de la precarización, la división social del trabajo y los atravesamientos de género. 

las patologías asociadas al trabajo contemporáneo y a la depreciación de la calidad del empleo. En ese sentido, la escuela de la psicodinámica del trabajo se propone una lectura crítica de las situaciones propias del trabajo posfordista particularmente recuperadas en los estudios locales que resignifican los aportes socio-históricos (Wlosko, 2015 ; Wlosko, 2017 ; Pujol, 2013 ; Pujol \& Gutiérrez, 2015) y que resaltan la generalización del miedo en los ámbitos organizacionales al afirmar que el miedo a la precarización constituye un verdadero sistema de management (Pujol, 2016)

Esta generalización del miedo constituye uno de los signos de época que Dejours (2006) resignifica en términos de patologías que caracterizan las nuevas formas de organizar el trabajo : las patologías de sobrecarga (física, cognitiva y emocional) y la servidumbre voluntaria que fuerza a los sujetos a evidenciar cuan adaptados e integrados se encuentran a la organización y cuan eficaz resulta su desempeño a la hora de dar respuesta a todo lo que le es requerido. En este marco se refuerza la lógica instrumental y la organización aprovecha la vulnerabilidad del trabajador para ganar aún más en productividad, siempre apoyándose en los preceptos del buen desempeño. Finalmente, también la violencia constituye un signo de época que al igual que en otros ámbitos de la vida social no remite solo al golpe físico sino al acoso moral, al maltrato psicológico y a la negación del otro (los pares, los colaboradores, los superiores) como interlocutor significativo.

Nuestros estudios sobre trabajo docente también resignifican supuestos sobre la salud alejados de las ideas tradicionalmente acuñadas por las instituciones internacionales y los enfoques de tradición local, que remiten a la noción de estado de salud, equilibrio o adaptación y enfatizan el papel de la actividad -social e históricamente situada- como contexto de desarrollo y el rol protagónico de los sujetos en la transformación de la actividad como motor en la conquista de la salud (Gutiérrez \& Dall' Asta, 2016; Gutiérrez, 2018). Se rompe así la distinción radical entre el estado de salud y el de enfermedad y se enfatiza en el espacio que entre ambas instancias posibilita la lucha contra la enfermedad, desde la cual es posible hablar de momentos de cierto equilibrio, pero estos -aun siendo satisfactorios o aceptables- siempre serán precarios y nunca ideales. Por fuera del ideal ubica entonces la noción de normalidad, refiriendo ella sí a un estado real.

Esta normalidad en línea con las contribuciones de Dejours excluye "la utopía de un trabajo sin sufrimiento" (Dejours, 1998b, p. 149) y remite a un estado de normalidad sufriente, que considera la normopatía derivada de los niveles de disciplinamiento y codificación que adquiere el trabajo contemporáneo (Wlosko, 2015 ; Pujol, 2018). Bajo el mismo supuesto y considerando que el trabajo no se produce en soledad se destaca la dimensión social y colectiva del trabajo, que se pone en línea con la dimensión social de la salud contenida en la psicodinámica del trabajo bajo el supuesto fundante de que no hay salud individual sino que esta se expresa a partir de la calidad del compromiso del sujeto en la relación con otros, en tanto mediadores que además enseñan o acompañan, por lo que la salud remite a una dinámica intersubjetiva.

42 En la medida en que la salud responde a una dinámica intersubjetiva, el trabajo como actividad tiene más de estructurante que de alienante. Los colectivos que se establecen en el trabajo serán entonces un territorio privilegiado para la construcción y defensa de la salud. En el campo del trabajo, la construcción de la subjetividad está ligada al reconocimiento y es por ello que la naturaleza, la calidad y la dinámica de las relaciones 
en los colectivos de trabajo tienen una responsabilidad considerable con respecto a los efectos del trabajo/empleo sobre la salud.

Las contribuciones de la psicosociología del trabajo (Lhuilier, 2006) y de la clínica de la actividad (Clot, 2008) ocupan un lugar clave para revalorizar la importancia que adquieren los propios sujetos del trabajo como protagonistas de las transformaciones y del trabajo como actividad, y la actividad humana como fuente de aprendizaje y desarrollo personal (Pujol, 2015, 2018).

Esta línea propone la distinción entre la tarea teórica imaginada por los organizadores del trabajo como fotografía congelada de lo que sucede en la situación de trabajo y la actividad real que los trabajadores emprenden, que se ofrece como resquicio para que el sujeto opere como protagonista de su propio desarrollo. Sin ese espacio para interpretar la prescripción, para (re)crear, la alienación es el único destino posible.

La actividad de trabajo está sustentada en un proyecto de transformación de lo real y de construcción de significados personales y sociales que jerarquiza el papel de los colectivos de trabajadores : Lo real implica un horizonte que va más allá de la realidad, entendida como estado de las cosas y se hace presente en particular en su dimensión de fracaso, a la vez que deriva en "un compromiso que contiene una dimensión de imaginación, de innovación, de invención" (Dejours, 1998. p. 43). En el mismo sentido se orienta Clot, alentando la exploración de las actividades excluidas, rechazadas o impedidas, incluso las contra-actividades, afirmando que "lo real de la actividad es incluso lo que no se hace, lo que no se puede hacer, lo que se intenta hacer sin lograrlo" (Clot, 2008, p. 89, traducción libre).

46 En tal sentido, Clot (2010) señala que restringir la acción a la estricta conservación del ser es un obstáculo para la salud y que la salud remite más claramente al desarrollo del poder de acción sobre el entorno y sobre sí mismo, mientras que las defensas que los trabajadores construyen para salvar su normalidad los rebajan, porque la normalidad no es más que la adopción de una norma única, mientras que la salud responde a la capacidad de producir nuevas normas, incluso en contra de la norma vigente.

El proceso de salud-enfermedad puede explicarse entonces de manera dinámica, como una tensión sostenida entre trabajo prescripto y trabajo real que el sujeto enfrenta a través del despliegue de estrategias colectivas de defensa, pero también de modo creativo a través de procesos de movilización subjetiva, de la puesta en juego de la inteligencia práctica.

48 La psicodinámica del trabajo plantea que en los procesos de movilización subjetiva se produce una inversión del cuerpo cognitivo-afectivo y la acción sobre lo real, con el fin de obtener logro y placer en la realización del trabajo. A su vez, del mismo modo que cada ámbito de actividad da lugar a particulares defensas ante el sufrimiento, también son específicos los anclajes en los que se inscriben las vivencias de placer.

En la reconstrucción de los procesos de subjetivación, entendemos importante identificar las características que adquiere la inteligencia práctica en los procesos concretos de trabajo, a fin de comprender sobre la base de qué apuntalamientos o a partir de qué recursos personales es posible pensar en la acción transformadora del sujeto sobre el malestar en ese ámbito concreto de trabajo. Asumimos el supuesto de que la movilización de la inteligencia práctica constituye una instancia generadora de placer porque su puesta en juego en una situación solo es posible actualizando la propia trayectoria de aprendizajes de manera creativa, y porque ese ajuste creativo también 
responde a lo compartido con el colectivo, en el sentido del género profesional (Clot, 2008) o en el sentido de acción tradicional eficaz (Dejours, 1998).

En este sentido, las conceptualizaciones más recientes de la psicodinámica del trabajo suponen al trabajador emocionalmente necesitado de operar en ruptura con relación al trabajo prescripto, y la clínica de la actividad lo concibe además cognitivamente dotado de recursos para hacerlo. En esta instancia el trabajador tiene la posibilidad de reflexionar sobre la acción y emprender una reconstrucción dinámica de la propia trayectoria vital identificando esos acontecimientos que han movilizado su subjetividad En el espacio de tensión entre la prescripción y la actividad se inscribirán entonces los aspectos capaces de generar salud, por la vía de posibilitar la movilización subjetiva y una dinámica que permita gestionar colectivamente el malestar.

51 Aunque la presencia de las contribuciones de la ergología no encuentra expresión todavía en las investigaciones locales, sus principales supuestos constituyen una vía regia para repensar distintas dimensiones de la intervención en las situaciones de trabajo. Para la ergología, la orientación clínica se construye a partir del deseo de conocer e intervenir en una situación de trabajo, reconociendo que los saberes puestos en juego por profesionales y trabajadores son de igual valor, diferentes pero no jerarquizados (Schwartz \& Durrive, 2009). Se trata de una construcción cooperativa, a partir de la escucha implicada respecto de lo que el trabajo impone a los trabajadores, de los conflictos psíquicos y sociales implicados, de las luchas del colectivo y de los desafíos que los trabajadores afrontan para mejorar sus vidas.

52 En este marco, se hace preciso capturar las singularidades de las situaciones desde la narrativa de los protagonistas considerando que se trata de un abordaje antropológico político y ético que entiende la actividad de trabajo como la imbricación del sujeto en un debate de normas, en el uso constante de sí, en una dramática que se constituye y se desarrolla en el cuerpo, en permanentes renormalizaciones (Schwartz \& Durrive, 2009) que a su vez exigen de astucia y creatividad.

En la comprensión de los procesos de innovación y su vinculación con la salud, la clínica de la actividad referencia a la importancia de la crisis de los saberes de oficio, de los saberes técnicos. En esta línea, se observa actualmente que existen serias dificultades en los grupos para conversar sobre los saberes acerca de la actividad y que el silencio sobre el propio quehacer en términos técnicos, el opacamiento de la construcción de los procesos de saber articulados o la pérdida de la cultura de conversaciones acerca del oficio, es otro de los elementos que afectan actualmente la salud : cuando el saber deja de estar en el centro, el sujeto también queda afectado en su salud de manera significativa.

54 La depreciación de los saberes técnicos acarrea una significativa pérdida de consistencia, de densidad de la palabra. La referencia a saber y conocer apela aquí al dominio y la reflexión sobre la acción, y también a la identificación, reconocimiento y apropiación de saberes ya producidos e incorporados a la cultura. Paradojalmente, en una economía del conocimiento, cada vez tenemos menos tiempo, menos espacio y menos grupos con quienes asumir la empresa de colectivizar saberes.

El potencial transformador de la realidad que reside en la actividad queda implicado en las deliberaciones sobre la calidad del trabajo: los trabajadores disponen de los recursos psicológicos y sociales para realizar un trabajo de calidad y discutir los criterios con los que es juzgado, por lo que en verdad preocupa menos a la clínica el trabajador expuesto a riesgos con su supuesta pasividad que la búsqueda de 
capacidades que ignoramos, en tanto la clínica se trata menos de promover el bienestar que de redescubrir con el trabajador el placer del bien-hacer (Clot \& Gollac, 2014), en tanto allí se encuentra uno de los resortes principales de la salud en el trabajo.

La clínica de la actividad singulariza en el campo del trabajo como la regeneración de un profesionalismo deliberado (Clot \& Gollac, 2014) que propicia la salud. Al respecto, se remarca que en el trabajo no es suficiente con ser reconocido por alguien sino reconocerse además en una historia común, un producto, una técnica, un lenguaje, una marca, un oficio, una trayectoria, sin lo cual resulta difícil ser alguien (Clot \& Gollac, 2014 ; Lhuilier, 2006).

En su propuesta de una psicología de los entornos de trabajo y vida, la clínica de la actividad resignifica el papel de la eficacia y el sentido en el desarrollo de la actividad de trabajo : trabajar es "lograr hacer lo que se quería hacer, ahorrando esfuerzos, pero también es juzgar lo que se hace en función de lo que se hubiera podido o debido hacer" (Clot, 2008, p. 89, traducción libre). Así, el sentido de la acción es el valor que el sujeto instaura entre dicha acción y sus otras actividades posibles y al hacerlo regula la actividad a través de múltiples recreaciones de los objetivos. En este marco, las controversias sobre el trabajo bien hecho resultan algo normal en la vida profesional y su negación, por el contrario, "intoxica las relaciones sociales y las desvitaliza" (Clot \& Gollac, 2014, p. 133, traducción libre). La clínica tiene por objetivo eliminar esta inhibición dialógica por la vía de retomar la creación profesional colectiva.

\section{Desafíos del desarrollo de una clínica del trabajo en Argentina}

58 El propósito central de esta revisión es poner en valor la capacidad heurística de las clínicas del trabajo como recursos potentes para tematizar las relaciones salud-trabajo en Argentina e intervenir sobre dimensiones no exploradas desde las perspectivas actualmente más utilizadas, en la medida en que se trata de una mirada crítica y emancipatoria que pone en discusión la hegemonía de los técnicos y los gestores en la definición y abordaje de la salud en el trabajo.

Esta posición supone una discusión ideológica, ética y política, y es en este marco en el que se plantean algunos de sus desafíos : por una parte, en lo referido a la producción de conocimientos, el desafío de la comprensión del trabajo contemporáneo, qué características asume, qué implicancias tiene para la salud, qué significa para los sujetos, desde una perspectiva comprensiva y crítica ; recuperando el carácter sociohistórico de los procesos de subjetivación.

En el campo de las intervenciones profesionales, entendemos necesaria una discusión sostenida acerca del rol de los profesionales en el abordaje del sufrimiento y el malestar y de la importancia de la co-construcción con los colectivos de trabajadores de saberes transformadores. En tal sentido, entendemos que la investigación-acción debe proyectarse en el plano de la organización del trabajo como instancia de configuración de colectivos implicados, con capacidad de agencia respecto de la conquista de la salud, como vía privilegiada para la construcción de entornos de trabajo que expandan las posibilidades de acción de los trabajadores y posibiliten su desarrollo.

61 Finalmente, en el ámbito académico y de la formación profesional, la perspectiva clínica abre a una propuesta de formación reflexiva, en territorio y en diálogo con los actores 
sociales, las instituciones y los pares (campo intelectual, empresarios, gobierno, sindicatos y trabajadores). Esta formación se halla necesariamente situada a partir de la agenda de los trabajadores, lo que contribuye a identificar las dimensiones idiosincráticas de una clínica del trabajo para nuestra región, de un abordaje que identifique cada vez con más claridad los modos en que el trabajo contemporáneo se expresa a nivel local y sus impactos en la salud; una clínica que disponga de capacidades para interpretar y poner en discusión los encargos sociales a la disciplina y las prácticas reproductivistas, e intervenir en un sentido transformador.

\section{BIBLIOGRAFÍA}

Borgeaud-Garciandía, N. (2016). Intimidad, sexualidad, demencias. Estrategias afectivas y apropiación del trabajo de cuidado en contextos desestabilizantes. Papeles del CEIC, 2016/1(148), CEIC (Centro de Estudios sobre la Identidad Colectiva). http://dx.doi.org/10.1387/pceic.15229

Cebey, M, \& Ferrari, L. (2010). Funciones, expectativas y riesgos psicosociales del trabajo contemporáneo. Anuario de investigaciones, 17, 91-98. Recuperado en 02 de octubre de 2019, de http://www.scielo.org.ar/scielo.php? script=sci_arttext\&pid=S1851-16862010000100009\&lng=es\&tlng=es.

Clot, Y. (2008). Travail et pouvoir d'agir. Paris : PUF.

Clot, Y. (2010). Travail au cœur : pour en finir avec les risques psychosociaux. Paris : La Découverte.

Clot, Y., \& Gollac, M. (2014). Le travail : peut-il devenir supportable? Paris : Armand Collin.

Dejours, C. (1998). El factor humano. Buenos Aires : Piette/Conicet.

Dejours, C. (2006). La banalización de la injusticia social. Buenos Aires : Topia.

Dejours, C. (1998b). Cómo formular una problemática de la salud en ergonomía y medicina del trabajo. In D. Dessors \& M. Guiho-Bailly (Comps), Organización del trabajo y salud (pp. 139-161). Buenos Aires : PIETTE/CONICET.

De la Garza-Toledo, E. (2009). Hacia un concepto ampliado de trabajo. In J. Neffa, E. De la GarzaToledo \& L. Muñiz-Terra (Comps.), Trabajo, empleo, calificaciones profesionales, relaciones laborales e identidades laborales (pp. 111-140). Buenos Aires : Grupos de Trabajo CLACSO.

Ferrari, L., Filippi, G., \& Trotta, M.F. (2011). ¿Moderadores o condicionantes estructurales del campo organizacional de emergencia del maltrato ? XVIII Anuario de Investigaciones. Volumen XVIII Tomo I. 117-123. Disponible en https://www.redalyc.org/pdf/3691/369139947022.pdf

Ferrari, L., Filippi, G., Novo, P., Sicardi, E., \& Trotta, M. (2013). La intervención frente a la violencia laboral desde la psicología del trabajo. Enfoques, perspectivas y experiencias. Revista E+E Estudios de Extensión en Humanidades, 3(3). Disponible en https://revistas.unc.edu.ar/index.php/ $\mathrm{EEH} /$ issue/view/469

Gutiérrez, I. (2018). Contribuciones de la psicodinámica del trabajo para el abordaje de la salud y seguridad en el trabajo : alcances y limitaciones. In O. Orozco-García, N. Del Castillo-Marín \& J. 
Román-Hernández (Eds.), Seguridad integral en el trabajo : un enfoque psicosocial (pp. 91-110). Cali, Colombia : Editorial Bonaventuriana.

Gutiérrez, I., \& Dall'Asta C. (2016). La actividad del docente de educación superior desde una perspectiva clínica. Identidad y salud. In J. Navarra. \& F. Barnes (Comps.), Cuadernos TAS : Trabajo, Actividad y Subjetividad. Escritos entre pares. Córdoba : Universidad Nacional de Córdoba.

Henry, M., \& Neffa, J. (2019). Riscos psicossociais no trabalho : diferentes visões para seu estudo no contexto argentino. Trabalho (En) Cena, 2019, V4(Especial), 01-06. DOI :

10.20873/2526-1487V4NEspecialP01

Kohen, J. (1997). Nuevas relaciones laborales y salud de los trabajadores en Argentina : desafíos y perspectivas para la investigación. Cadernos de Saúde Pública, 13 (Suppl. 2), 47-57. https:// dx.doi.org/10.1590/S0102-311X1997000600005

Lhuilier, D. (2006). Cliniques du travail. Paris : Editions Érès. https://doi.org/10.3917/eres.lhuil. 2007.01

Neffa, J. (1995). Las condiciones y medio ambiente de trabajo (CyMAT). Presentación de la concepción dominante y de una visión alternativa. Buenos Aires : Serie CyMAT, PIETTE-CONICET.

Neffa, J. (2015). Los riesgos psicosociales en el trabajo : contribución a su estudio. Buenos Aires : CEILCONICET.

Neffa, J., \& Del Bono, A. (2016). Una visión de conjunto sobre los estudios del trabajo en Argentina (1990-2014). In E. De la Garza-Toledo (Ed.), Los estudios laborales en América Latina. Orígenes, desarrollos y perspectivas. (pp. 36-75). México : Anthropos-UAM.

Paugam, S. (2006). Les fragmentations de la société salariale. Un autre regard sur la structure sociale. Sociologia : Revista da Faculdade de Letras da Universidade do Porto, 16, 161-176. https:// www.redalyc.org/articulo.oa ?id =4265/426539975010

Pujol, A. (2013). La qualité du travail au secteur TI en Argentine. Les tensions entre les trajectoires singulières et collectives. Nouvelle Revue de Psychosociologie, (15), 131-144. https:// doi.org/10.3917/nrp.015.0131

Pujol, A. (2015). Travail créatif et approche clinique. Education Permanente (dossier Travail, créativité, subjectivité), (202), 53-64. Disponible en : http://www.education-permanente.fr/public/ articles/articles.php?id_revue=1733\&id_article=2410\#resume2410

Pujol, A. (2016). La violencia laboral en la universidad. Desafíos para la política gremial. Revista Trabalho (En)Cena, 1(1), 99-116. Disponible en : https://sistemas.uft.edu.br/periodicos/index.php/ encena/article/view/2270

Pujol, A. (2018). La organización del trabajo como categoría estructurante de la salud y seguridad en el trabajo. In O. Orozco-García, N. Del Castillo-Marín \& J. Román-Hernández (Comps.), Seguridad integral en el trabajo. Un enfoque psicosocial (pp. 57-76). Cali : Editorial Bonaventuriana.

Pujol, A., \& Gutiérrez, I. (2015). Violencia Laboral : Nuevos escenarios institucionales y encrucijadas del abordaje clínico. In L. Godoy \& E. Ansoleaga, E. (Comps.) Un campo en tensión o tensión entre campos. Psicología de las organizaciones y del trabajo en Iberoamérica (pp. 361-374). Santiago de Chile : Ril editores.

Pujol, A., Gutiérrez, I., \& Barnes, F. (2015). Cuidar y cuidarse en el trabajo. Contribuciones desde una perspectiva clínica. In A. Pujol \& I. Gutiérrez (Comps.), Trabajo y subjetividad. Indagaciones clínicas (pp. 117-132). Doi : http://hdl.handle.net/11086/2323 
Schwartz, Y., \& Durrive, L. (2009). L'activité en dialogues. Entretiens sur l'activité humaine (II). Toulouse : Octarès Editions.

Wlosko, M. (2015). Clínica de los procesos subjetivos en relación al trabajo : el abordaje de la Psicodinámica del Trabajo. VERTEX Revista Argentina de Psiquiatría, 16, 417-426. Disponible en http://www.polemos.com.ar/docs/vertex/vertex124.pdf\#page $=18$

Wlosko, M. (2017). Métodos de organización del trabajo, racionalidad neoliberal y Salud Mental. Revista Salud Mental y Comunidad, 4(4), 95-99

Wlosko, M., \& Ros, C. (2009). Violencia laboral : hipótesis y niveles explicativos a la luz del caso del personal de enfermería del sector público. In S. Slapak (Presidencia), I Congreso Internacional de Investigación y Práctica Profesional en Psicología, XVI Jornadas de Investigación, Quinto Encuentro de Investigadores en Psicología del MERCOSUR. Conferencia llevada a cabo en Facultad de Psicología, Buenos Aires. Disponible en : https://www.aacademica.org/000-020/306

Wlosko, M., \& Ros, C. (2012). Violencia Laboral en el sector salud : Abordajes conceptuales y resultados de investigación en personal de enfermería en la Argentina. In E. Ansoleaga-Moreno, O. Artaza-Barrios \& J. Suárez-Jiménez (Comps.), Personas Cuidando Personas : Ser Humano y Trabajo en salud (pp. 211-230). Santiago : OPS/OMS.

\section{RESÚMENES}

El artículo reconstruye brevemente las perspectivas de abordaje de las relaciones salud y trabajo que contribuyen a la comprensión de la problemática de la salud laboral en Argentina. En el desarrollo se consideran de modo estilizado sus principales supuestos y orientaciones; posteriormente considera aportes de la clínica del trabajo que discuten algunos principios que orientan estas intervenciones, sus alcances y desafíos. El análisis se posiciona en la clínica del trabajo -que asume en Argentina carácter emergente- ; destaca los aportes que la diferencian de los enfoques predominantemente en uso tanto en el plano de las proposiciones de base como en sus derivaciones metodológicas. Como cierre, se esbozan algunos desafíos de la clínica del trabajo en nuestro campo disciplinar que, de ser asumidos, permitirán enriquecer los debates y prácticas actuales de la psicología en torno a las tensiones que se expresan en el plano de la salud en los actuales escenarios laborales.

O artigo reconstrói brevemente as perspetivas de abordagem das relações entre saúde e trabalho que contribuem para a compreensão da problemática da saúde ocupacional na Argentina. As suas principais suposições e orientações são apresentadas de forma estilizada, para posteriormente considerar contribuições da clínica de trabalho que discutem alguns princípios que norteiam essas intervenções, seus alcances e desafios.

A análise está posicionada na clínica do trabalho - que assume um caráter emergente na Argentina - e destaca as contribuições que a diferenciam das abordagens predominantemente em uso, tanto em termos das propostas básicas quanto em suas derivações metodológicas.

Como conclusão, são delineados alguns desafios da clínica do trabalho no nosso campo disciplinar que, se assumidos, permitirão enriquecer os debates e práticas atuais da psicologia em torno das tensões que se expressam no plano da saúde nos cenários atuais de trabalho.

L'article décrit brièvement les approches de la santé au travail qui contribuent à la compréhension du problème en Argentine. Leurs principales hypothèses et orientations sont présentées de manière stylisée pour considérer les contributions de la clinique du travail qui mettent en discussion les principes guidant ces interventions. L'analyse se situe dans la clinique du travail - qui revêt un caractère émergent en Argentine - et met en évidence les contributions 
qui le différencient des approches plus utilisées, à la fois en termes de propositions de base et de leurs dérivées méthodologiques. En guise de conclusion, certains défis de la clinique du travail dans notre domaine disciplinaire sont décrits que s'ils sont assumés, permettront d'enrichir les débats et les pratiques psychologiques actuelles autour des tensions exprimées sur le plan de la santé dans les scénarios de travail actuels.

The paper briefly reconstructs the perspectives of approach to the relationship between health and work that contribute to understanding the problem of occupational health in Argentina. In the discussion, their main assumptions and orientations are considered in a stylized way; subsequently, contributions from the work clinic are considered, discussing some principles that guide these interventions, their scope and challenges. The analysis is positioned in the work clinic -which is just emergent in Argentina- highlighting the contributions that differentiate it from predominant approaches, both at the basic proposals and at its methodological derivations. As a closing, some challenges of the work clinic in our disciplinary field are outlined; if assumed, they will enrich the debates and current practices of psychology about the tensions that are finding expression in the health area at current work scenarios.

\section{ÍNDICE}

Keywords: health, work, work clinic, Argentina

Palabras claves: salud, trabajo, clínica del trabajo, Argentina

Mots-clés: santé, travail, clinique du travail, Argentine

Palavras-chave: saúde, trabalho, clínica do trabalho, Argentina

\section{AUTORES}

\section{ANDREA PUJOL}

Facultad de Psicología de la Universidad Nacional de Córdoba, Argentina - Deán Funes 1963, Dpto. 14, Córdoba, Argentina - pujol.andrea@gmail.com

\section{MARÍA INÉS GUTIÉRREZ}

Facultad de Psicología de la Universidad Nacional de Córdoba, Argentina - Baden Powell 2943, Córdoba, Argentina - gutierrezmariaines@gmail.com 